\title{
Synthesis of Gold Nanorods for Biomedical Applications
}

\author{
M.A. García ${ }^{\mathrm{a}}$ V. Bouzas ${ }^{\mathrm{b}}$, and N. Carmona ${ }^{\mathrm{b}}$ \\ ${ }^{a}$ Glass and Ceramic Institute. Spanish Council for Scientific Reseach. C/ Kelsen, 528049 Madrid \\ (Spain) \\ ${ }^{b}$ Materials Physics Department. Complutense Univesity at Madrid. Avda. Complutense s/n, 28040 \\ Madrid (Spain)
}

\begin{abstract}
Gold nanoparticles are commonly used in biomedical applications due to their high biocompatibility and easy modular functionality. Their unique optical properties, such as Surface Plasmon Resonance (SPR), lead to a great light absorption in the visible range of the spectrum. Here, we show that the synthesis of non spherical gold nanoparticles causes the maximum absorption band to shift to the near-infrared region of the spectrum, thus avoiding overlaps with other biological tissues
\end{abstract} (eg. Haemoglobin in bloodstream).

Keywords: Nanoparticles, gold nanorods, synthesis, surface plasmon resonance, optical properties.

PACS: 78.67.Bf (nanocrystals, nanoparticles and nanoclusters), 78.67.Qa (Optical properties of nanorods), 81.07.Bc (nanocrystalline materials: fabrication and characterization).

\section{GOLD NANOPARTICLES AND NANORODS}

Surface plasmon resonance (SPR) is the most outstanding feature of metallic nanoparticles (NPs). It consists on a collective oscillation of the conduction electrons inside the NPs. The resonant frequency of noble metal nanoparticles specifically, falls in the visible part of the spectrum ${ }^{1}$. Therefore, when illuminating noble metal with visible light, surface plasmons (SP) can be excited leading to a great light absorption even with very small quantities of nanoparticles.

The SPR in gold nanoparticles is so strong that it enhances locally the light electric field several orders of magnitude. Actually, the cross-section of the absorption associated with the SP excitation can be up to 1000 times larger than its geometrical section $^{2}$. These effects, as well as the high biocompatibility and easy modular functionality rend gold nanoparticles as attractive elements for biomedical applications $^{3-4}$.

The main disadvantage for the use of Au nanoparticles in biomedicine is due to the fact that the SPR absorption band matches that of haemoglobin one, rending a huge task to optically detect Au nanoparticles in bloodstream (Fig. 1). This limitation can be 
overcome by the synthesis of non spherical Au nanoparticles, as nanorods, for which the SRP splits up into two bands, one associated with the SP excited longitudinal rod axis and the other to the transversal. The first absorption band falls approximately in the same position that SPR of spherical nanoparticles ( $525 \mathrm{~nm})$, the second one shifts toward larger wavelengths. Consequently, tailoring the aspect ratio (length/width) of the NRs enables to shift the perpendicular SPR to higher wavelengths of the spectrum (750-1200 nm) (to Near-infrared region, NIR) where the biological tissues and blood scarcely absorbs. Therefore, it would be possible to excite SRP in vivo.

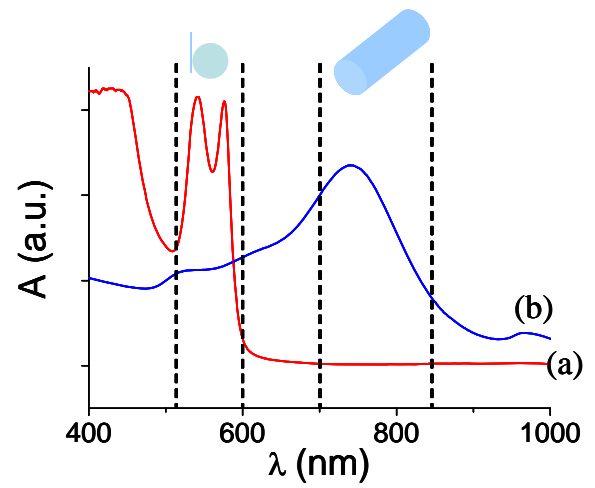

FIGURE 1. Optical absorption spectra of: a) blood and b) Au NPs. Vertical lines show the absorption range of Au NSs and NRs.

\section{SYNTHESIS: SEED-MEDIATED GROWTH METHOD}

One of the most simple and reproducible methods to synthesize Au nanorods is the seed-mediated growth method ${ }^{5-7}$. It consists of a two step process, i.e. nucleation and then successive growth of the NPs. By introducing some modifications in the preparation conditions (eg. carefully control of the solutions temperature, stirring, ratio of seed to metal salt, etc...) we were able to obtain a defined aspect ratio of the Au NRs.

In the first step, a growth solution is prepared by the mild reduction of $\mathrm{Au}$ ions ( $\mathrm{HAuCl}_{4}$ 0.0010M) with L-ascorbic acid (0.0778M) in presence of $\mathrm{AgNO}_{3}(0.0400 \mathrm{M})$ which promotes anisotropy and (Hexadecyltrimethylammonium Bromide) CTAB $(0.20 \mathrm{M})$ and (Benzyldimethylhexadecylammonium Chloride) BDAC (0.15M) as surfactants. The seed solution is prepared afterwards by rapid reduction of the $\mathrm{Au}$ precursor with a strong reducing agent $\left(\mathrm{NaBH}_{4} 0.010 \mathrm{M}\right)$ in the presence of CTAB $(0.20 \mathrm{M})$ as surfactant. The final step is the addition of an aliquot of the seed solution to the growth one. 


\section{TUNING SPR POSITION}

Figure 2 shows the optical absorption spectra and the corresponding transmission electron microscopy images of syntheses performed at different experimental conditions. The shape and size of the Au NPs during each preparation have influence in their optical properties. Figure 2a shows the sole presence of Au NSs with a maximum absorption band at $540 \mathrm{~nm}$. In general, stirring during the synthesis favours homogeneity in the solution thus resulting in shorter and nearer to spherical shape NPs. Figures $2 \mathrm{~b}$ and c show two absorption bands centred at 552 and $530 \mathrm{~nm}$ respectively associated with the transversal SPR of the NRs and the SPR of spherical NPs that can result from the synthesis. Bands at 772 and $802 \mathrm{~nm}$ correspond to the longitudinal SPR of Au NRs. Optimization of experimental conditions result in the sole formation of Au NRs with a maximum absorption band at around $735 \mathrm{~nm}$ and high values of the absorption intensity (Fig. 2d). The position of the red-NIR band is adequate for the optical detection of the synthesized Au NRs ${ }^{8}$.
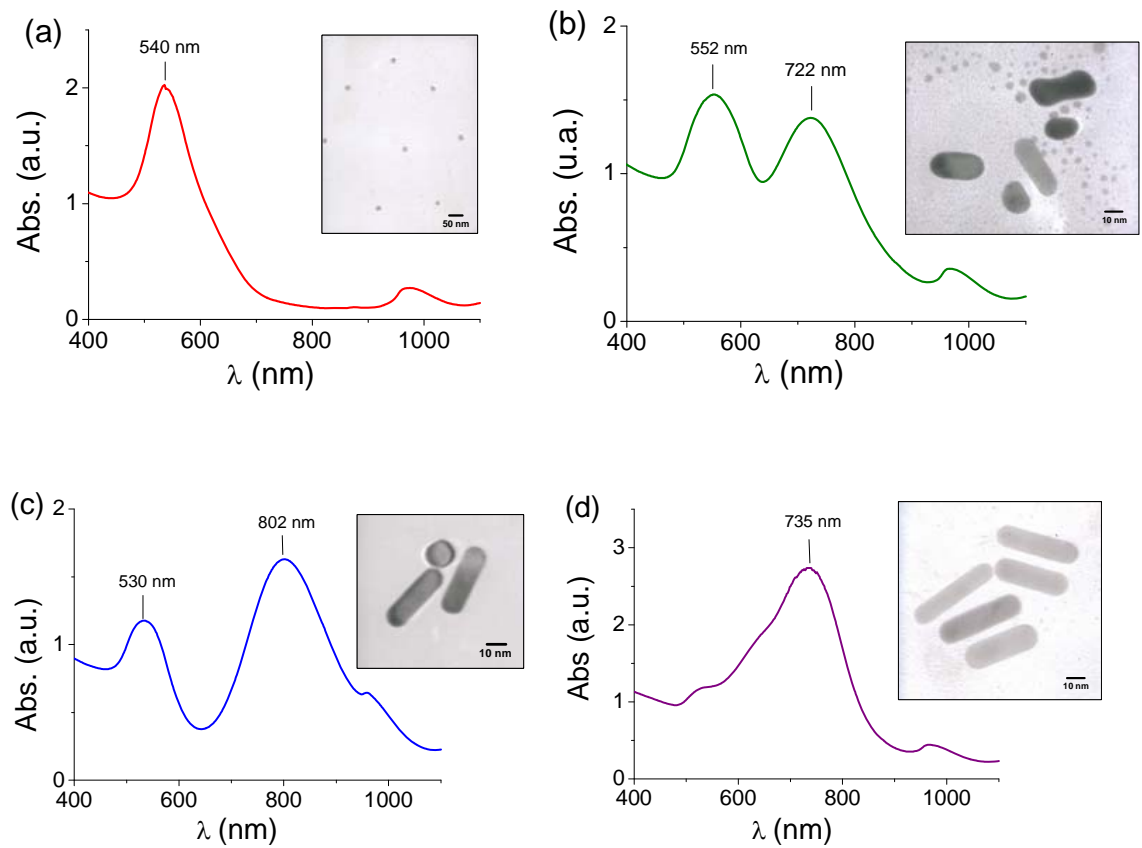

FIGURE 2. Optical absorption spectra and transmission electron microscopy images corresponding to synthesis of: a) Au NSs, b) and c) Au NSs and NRs with different aspect ratio, and d) Au NRs. 


\section{CONCLUSIONS}

Gold NRs were synthesized by the seed-mediated growth method. Adding some modifications and by carefully control of the preparation conditions the aspect ratio of the Au NPs was tuned. Optimal optical properties were found when the SPR was shifted to the NIR region with high absorption intensity and absence of Au NSs as residual product. Future work includes the design and preparation of a miniaturized prototype to detect and measure the Au NRs in bloodstream.

\section{ACKNOWLEDGMENTS}

Authors acknowledge the financial support from EU project BONSAI LSHBCT-2006-037639. NC acknowledges the financial support of the EC-MEC Ramón y Cajal Program RYC-2007-01715. Authors acknowledge Prof. J. Llopis (UCM) for support and help with the experimental work.

\section{REFERENCES}

1. J. A. Creighton and D. G. Eadon, J. of the Chem. Soc. Faraday Trans. 87 38813891 (1991).

2. N. Harris, M. J. Ford, P. Mulvaney, and M. B. Cortie. Gold Bulletin 41 5-14 (2008).

3. M. Hu, J.Chen, Z.Y. Li, L. Au, G.V. Hartland, X. Li, M. Marquez, and Y. Xia, Chemical Society Reviews 35 1084-1094 (2006).

4. L. Tong, Q. Wei, A. Wei, and J-X. Cheng, Photochemistry and Photobiology 85 2132 (2009).

5. N.R. Jana, L. Gearheart and C.J. Murphy, J. Phys. Chem. B $1054065-4067$ (2001).

6. B. Nikoobakht and M.A. El-Sayed, Chem. Mater. 15 1957-1962 (2003).

7. N. R. Jana, L. M. Liz-Marzán and C. J. Murphy, J. of Phys. Chem. B 105 4065-4067 (2001).

8. 1. See in this volume Miniaturized Devices For Au Nanorods Detection by M.A. García et al. 